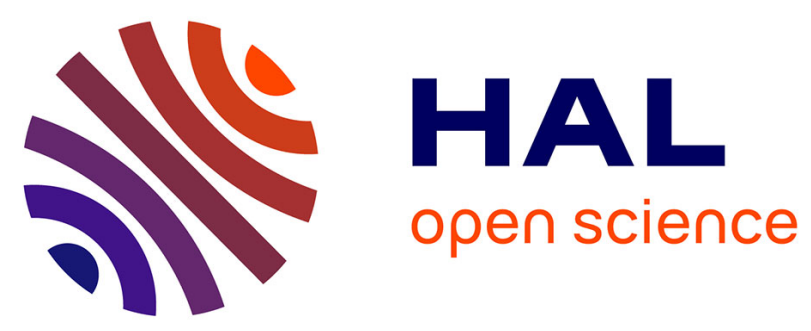

\title{
Soft sensor design for power measurement and diagnosis in electrical furnace: A parametric estimation approach
}

Baya Hadid, Erik Etien, Régis Ouvrard, Thierry Poinot, Laurent Le Brusquet, Anne Grau, Gilbert Schmitt

\section{- To cite this version:}

Baya Hadid, Erik Etien, Régis Ouvrard, Thierry Poinot, Laurent Le Brusquet, et al.. Soft sensor design for power measurement and diagnosis in electrical furnace: A parametric estimation approach. IECON 2013, Nov 2013, Vienne, Austria. pp.2191 - 2196, 10.1109/IECON.2013.6699471 . hal-00932274

\section{HAL Id: hal-00932274 \\ https://hal-centralesupelec.archives-ouvertes.fr/hal-00932274}

Submitted on 18 Nov 2014

HAL is a multi-disciplinary open access archive for the deposit and dissemination of scientific research documents, whether they are published or not. The documents may come from teaching and research institutions in France or abroad, or from public or private research centers.
L'archive ouverte pluridisciplinaire HAL, est destinée au dépôt et à la diffusion de documents scientifiques de niveau recherche, publiés ou non, émanant des établissements d'enseignement et de recherche français ou étrangers, des laboratoires publics ou privés. 


\title{
Soft Sensor Design for Power Measurement and Diagnosis in Electrical Furnace: a Parametric Estimation Approach
}

\author{
Baya Hadid*, Erik Etien*, Régis Ouvrard*, Thierry Poinot*, Laurent Le Brusquet ${ }^{\dagger}$, Anne Grau ${ }^{\ddagger}$ and Gilbert Schmitt ${ }^{\ddagger}$ \\ *Laboratoire d'Informatique et d'Automatique pour les Systèmes EA6315 (LIAS), \\ Université de Poitiers, 86000 Poitiers Cedex, France. \\ Email: (baya.hadid, erik.etien, regis.ouvrard, thierry.poinot)@univ-poitiers.fr \\ ${ }^{\dagger}$ Supélec Sciences des Systèmes - EA4454 (E3S). 3, rue Joliot Curie, 91192 Gif-sur-Yvette, France. \\ Email: Laurent.Lebrusquet@supelec.fr

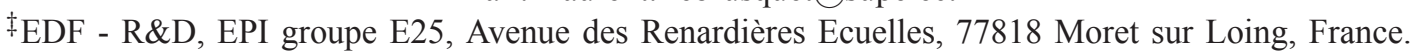 \\ Email: retd-epi-chic@edf.fr
}

\begin{abstract}
In this paper, we propose as a first step a software solution to measure the electrical power consumed in an industrial furnace intended essentially for heat treatments. The soft sensor is constructed from the power physical measurement taken as the output of the set (dimmer + resistances), and the control signal measurement provided by a controller with an unknown structure. The second step consists in a detection of faults like a resistance disconnection, for instance. This phase requires the knowledge of the controller model and the furnace system. An overparametrization method was chosen for the controller estimation. An indirect closed-loop Input-Output (IO) identification approach was used for the furnace model estimation through a Tailor-Made and a decomposition of the closed-loop algorithms. A validation with two other experimental tests concludes the paper.
\end{abstract}

\section{INTRODUCTION}

In France, the energy consumption of the industrial sector represents roughly $28 \%$ of the french energy consumption and $23 \%$ of its total $\mathrm{CO}_{2}$ emissions [8]. This situation is becoming unacceptable in a context of increasingly demands with respect to energy efficiency of industrial and household uses, especially when a great part of this energy can be saved.

That is in this context that the project ANR CHIC (CHaines de mesures Innovantes à bas Coût) emerged. The objective is to develop and test low-cost sensors to monitor and analyze the energy consumption of major fluids used in industrial sites (electricity, gas, compressed air). These sensors should allow the consumption monitoring and the detection of consumption deviations. The objectives are to achieve a measurement accuracy of about $5 \%$. The project involves the design of new low-cost sensors (both physical and software sensors) in the following areas: current sensors, voltage, power, and gas flow. The work presented in this paper only concernes the study of power measurement.

The objective of the study presented in this paper is, in a first step, to design of a power "soft" sensor. The concept of soft sensors is to combine measurement available or easily achievable, representing the evolution of the studied process, and mathematical models relating the measure and the quantities to determine. This concept is used in different fields and especially in chemical or biological processes [2]. Modeling is a key step to determine the quality of the measurement and can be based on a physical or empirical approach, or a combination of both (grey box models). The design of a physical model is excluded since it is poorly adapted to an industrial environment. For this purpose, we propose to build behavioral models.

The second step consists in a diagnosis of faults usually noticed in this kind of equipment. When it is the case, an overshoot in power is observed and may be caused by a door that was poorly closed or by a failure in one of the resistances of the furnace. The diagnosis procedure depends on the kind of occurred failure. We will see that a failure presence can be detected only with the control signal. Therefore, it is important to have a good estimation of the controller. However, this criterion is not sufficient in the sense that we can not distinguish what sort of failure has occurred. In this case, the study of the furnace model parameters such as its gain and time constants is essential.

The I/O furnace modeling in a closed-loop working environment was rarely discussed [1], [13]. There are two types of identification in such case: identification in open-loop and identification in closed-loop. The first one is in practice difficult or simply not feasible. For example, some plants have an integrator or are instable in open-loop, and others can undergo long term drift in open-loop operation and non-stationary disturbances which favor a closed-loop experiment for data collection [7]. Also, it has been shown that a closed-loop identification experiment is often the optimal experimental setup [3]. In the remainder of this paper, two different outputerror algorithms are discussed. 


\section{PLANT DESCRIPTION}

The furnace, shown in figure1, contains a two zones refractory brick chamber. Each zone includes 6 resistances $(12 \mathrm{~kW}$, $380 \mathrm{~V}$ ) which leads to $144 \mathrm{~kW}$ of total power. The temperature in each zones is regulated by a digital controller actuated by a dimmer following a setpoint profile imposed by the operator (figure 2), and measured by a thermocouple. Measurements (electric power $[\mathrm{kW}]$, furnace temperature $\left[{ }^{\circ} \mathrm{C}\right]$, control signal [\%]) are collected each $10 \mathrm{~s}$. The closed-loop scheme is summarized in the figure 3 . The maximum temperature is of $1600^{\circ} \mathrm{C}$.

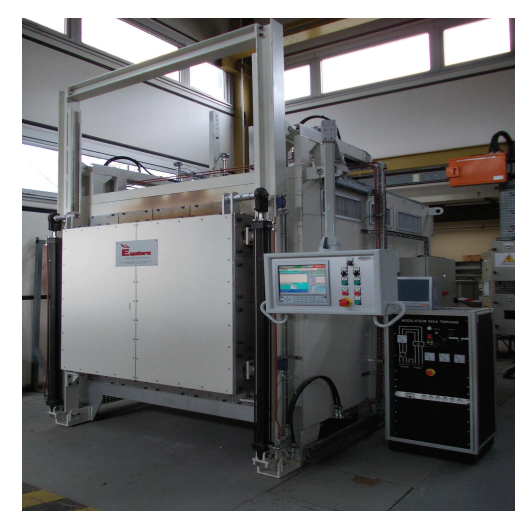

Fig. 1. Experimental plant

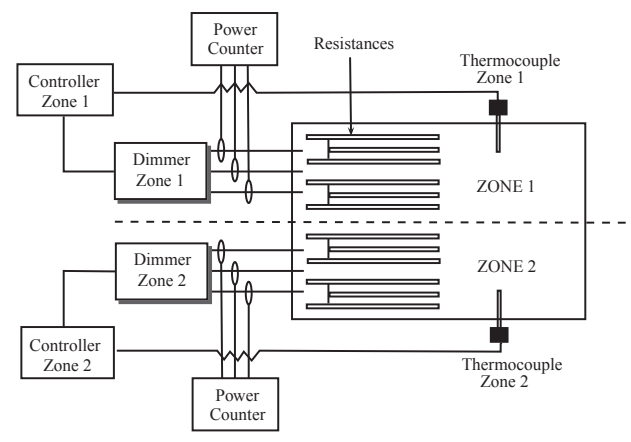

Fig. 2. Furnace rig

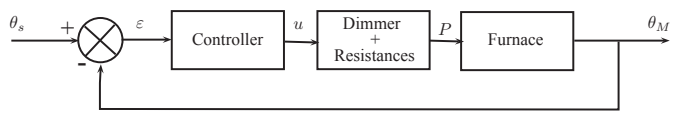

Fig. 3. Closed-loop system

The figure 4 presents some of the data collected on the furnace during a free-load common test. We can see that power is strongly related to the control signal.

\section{POWER MEASUREMENT}

The power soft sensor development comes down to model the set dimmer and resistances defined by the relationship $P=$ $f(u)$ between the power and the control signal as shown in the figure 3.
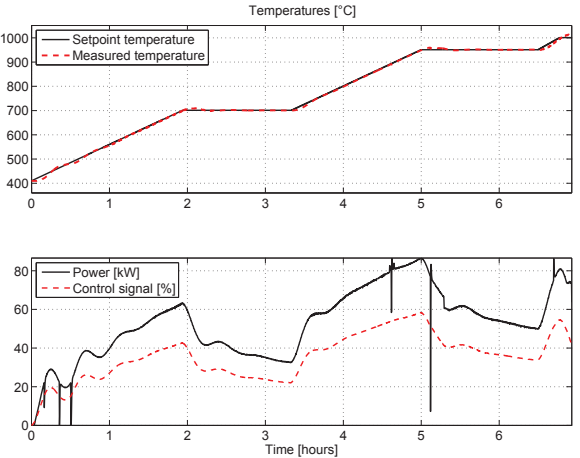

Fig. 4. Typical experimental data

Knowing that the furnace is of power equal to $144 \mathrm{~kW}$ and that the control signal varies between 0 and $100 \%$, the theoretical model of the dimmer and resistances is then deduced: $P(t)=1,44 u(t)$. The plot (Figure 5) of the experimental data $P=f(u)$ reveals a linearity. Thus, a simple leastsquares algorithm $(L S)$ is sufficient to model this relationship and gives : $P(t)=1.506 u(t)-0.9117$. Figure 5 shows the measured and simulated output. The figure 6 represents a

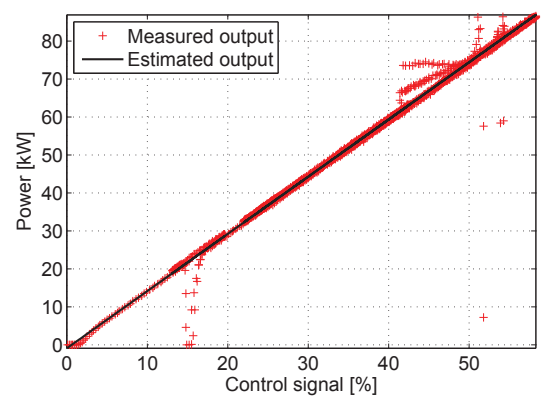

Fig. 5. Least-squares dimmer/resistances model estimation

comparison between the theoretical model and the LS-model. To verify the consistency of the estimated model, a validation was done on another experimental test as shown in the figures 7 and 8. The calculation of the relative error (see table I), without taking into account the outlier points, shows that its maximum is less than the fixed objective of $5 \%$ with a clearly advantadge for the LS-model.

\begin{tabular}{|c||c|c||c|c|}
\hline Test & $\operatorname{mean}_{L S}$ & $\operatorname{mean}_{T h}$ & $\max _{L S}$ & $\max _{T h}$ \\
\hline Estimation & 0.22 & 3.38 & 9.68 & 9.90 \\
\hline Validation & 0.22 & 3.29 & 9.75 & 9.96 \\
\hline \multicolumn{5}{|c}{ TABLE I } \\
\hline
\end{tabular}

MEAN AND MAXIMUM RELATIVE ERROR WITH ESTIMATION AND VALIDATION DATA

The interest of the estimated model is its simplicity to handle. It can be easily implemented with a microcontroller, for instance. It also gives the possibility to calibrate or recalibrate the model online.

\section{FAULT DIAGNOSIS}

An overshoot in power may be caused by a door that was poorly closed or by a fault in one of the resistances of the 

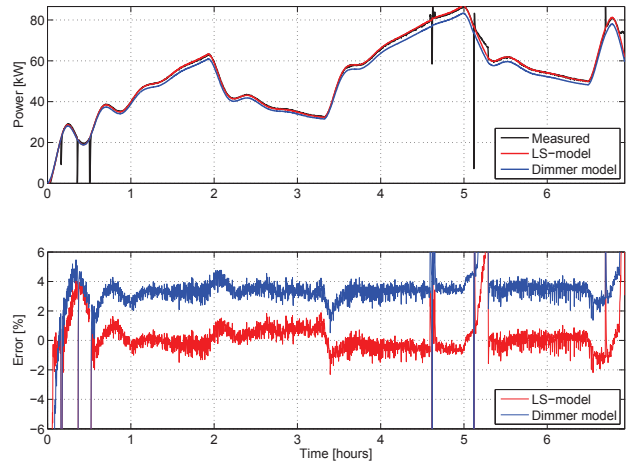

Fig. 6. Comparison between the estimated LS model output and the theoretical dimmer/resistances model output

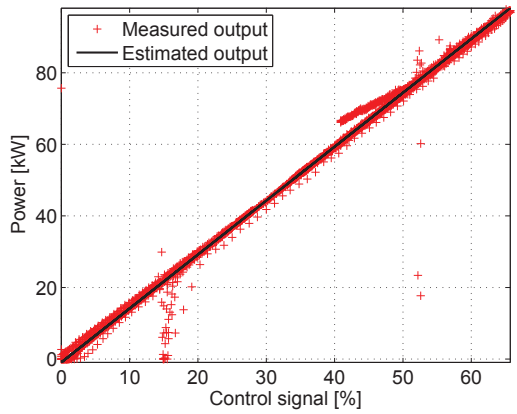

Fig. 7. Validation of LS dimmer/resistances model estimation
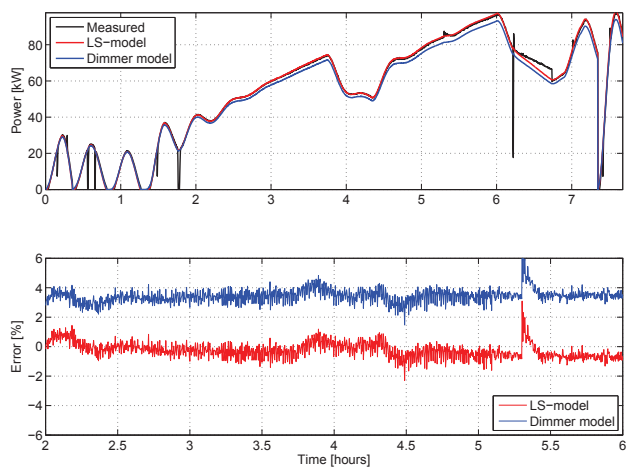

Fig. 8. Comparison between the LS-model output and the theoretical dimmer/resistances model output with validation data

furnace. Early detection of these faults can significantly reduce energy consumption by alerting the system operator.

As it has been said in introduction, this step involves the identification of the controller and the furnace system. Indeed, the feedback is taken into acount by identifying a closed-loop transfer function and determining the normal-operating using the knowledge of the linear controller.

\section{A. Controller estimation}

It might be obvious to know exactly the control law, particularly when the systems are numerically controlled. But in practice, the implemented controller can be very different from the real one, because of programming mode, nonlinearities, antisaturation devices, etc. A solution consists to identify the controller. In order to avoid the choice of the controller model, an overparametrization technique is used. Then a criterion based on discrete moment is used to choose the model order [12].

Consider the system given by the closed-loop setting of the figure 9. $y_{k}$ is the measurement of $x_{k}$ added to measurement

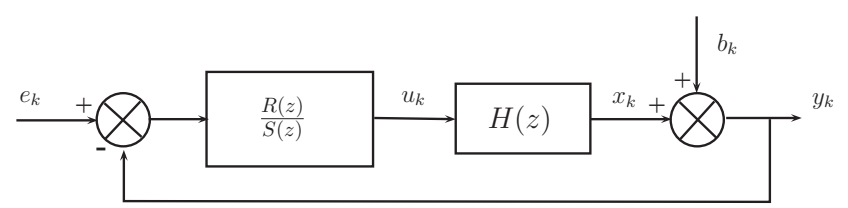

Fig. 9. Closed loop

noise $b_{k}\left(y_{k}=x_{k}+b_{k}\right.$ with $x_{k}$ unknown). Once the measurement is obtained, $y_{k}$ is well known. Thus, $y_{k}$ is a certain value that we can use in a processing control algorithm. The controller identification is therefore a free-noise identification problem. With the prior knowledge of the control signal $u_{k}$ and the setpoint $e_{k}$, we can apply an ordinary least-squares algorithm [9].

Let us define for example the controller to be estimated by

$$
\frac{R(z)}{S(z)}=\frac{r_{0}+r_{1} z^{-1}}{1+s_{1} z^{-1}}
$$

Then

$$
u_{k}+s_{1} u_{k-1}=r_{0}\left(e_{k}-y_{k}\right)+r_{1}\left(e_{k-1}-y_{k-1}\right)
$$

Knowing the control signal, the setpoint and the noisy output, we can write

$$
\hat{u}_{k}=\underline{\varphi}_{k}^{T} \underline{\hat{\theta}}
$$

with $\frac{\varphi^{T}}{T_{k}}=\left[\begin{array}{lll}e_{k}-y_{k} & e_{k-1}-y_{k-1} & -u_{k-1}\end{array}\right]$ and $\underline{\hat{\theta}}^{T}=\left[\begin{array}{lll}\hat{r}_{0} & \hat{r}_{1} & \hat{s}_{1}\end{array}\right]$.

If the exact structure is unknown, an overparametrization principle can be used. Thus, $C_{s}(z)=\frac{R_{s}(z)}{S_{s}(z)}$ is chosen such as

$$
\operatorname{deg}\left[R_{s}(z)\right]>\operatorname{deg}[R(z)], \operatorname{deg}\left[S_{s}(z)\right]>\operatorname{deg}[S(z)]
$$

Consider the overparametrized estimated controller

$$
C_{s}(z)=\frac{r_{0}+r_{1} z^{-1}+\ldots+r_{2} z^{-S}}{1+s_{1} z^{-1}+\ldots s_{S} z^{-S}}
$$

where $S$ is the degree of overparametrization, and $\hat{u}_{k}=$ $\underline{\varphi}_{S k}^{T} \underline{\hat{\theta}}_{S}$, with

$$
\left\{\begin{array}{l}
\varphi_{S k}^{T}=\left[\begin{array}{ll}
e_{k}-y_{k} \ldots e_{k-S}-y_{k-S}-u_{k-1} \ldots-u_{k-S}
\end{array}\right] \\
\underline{\hat{\theta}}_{S}^{T}=\left[\begin{array}{ll}
r_{0} \ldots r_{S} & s_{1} \ldots s_{S}
\end{array}\right]
\end{array}\right.
$$

It is necessary to be sure that the $S$ degree is sufficiently high in order to avoid modeling error and at the same time not uselessly high. For that purpose, a characterization test based on system invariants called "discrete moments" is used [15].

Let us define the controller impulse response $g_{k}$ with a finite sum in $\left[0, \infty\left[\right.\right.$. A Taylor series expansion of $C_{S}(z)$ in neighborhood of $z^{-1}=1$ gives 


$$
Z\left\{g_{k}\right\}=C_{S}(z)=\sum_{n=0}^{\infty} \frac{\left(z^{-1}-1\right)^{n}}{n !} M_{n}\left(g_{k}\right)
$$

where $M_{n}\left(g_{k}\right)=\sum_{k=n}^{\infty} A_{k}^{n} g_{k}$ with $A_{k}^{n}=\frac{k !}{(k-n) !}$, is the $n^{t h}$ order discrete moment of the impulse response $g_{k}$. In the case of a controller with an integrator, we deduce the moments of order 0,1 and 2 as follows

$$
\left\{\begin{array}{l}
M_{0}=\beta_{0} / \alpha_{1} \\
M_{1}=\left(\beta_{1}-C_{0} \alpha_{2}\right) / \alpha_{1} \\
M_{2}=2\left(\beta_{2}-C_{0} \alpha_{3}-C_{1} \alpha_{2}\right) / \alpha_{1}
\end{array}\right.
$$

with $\beta_{n}=\sum_{k=n}^{M} A_{k}^{n} r_{k}, \alpha_{n}=\sum_{k=n}^{N} A_{k}^{n} s_{k}$ and $M, N$ the numerator and denominator orders.

If an overparametrized structure includes the exact structure, then all its moments are equivalent to those of the real system. In practice, the exact moments are unknown, but we can increase the structure complexity and calculate its moments. When a stability of moments is reached, the considered structure includes certainly the real system.

This algorithm is now applied to the experimental data. Because of the lack of knowledge about this structure, an overparametrization to a degree $S=3$ was done. In order to find the good $S$ degree, the discrete moments are tested to find the degree from which the system invariants does not change as shown in table II. We can clearly observe a negligible variation of the discrete moment values for models higher or equal to $S=2$. Consequently, the estimated controller has the

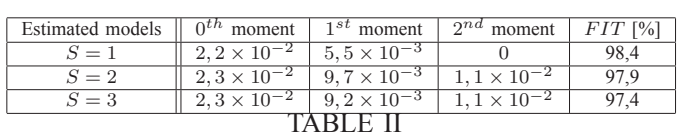

DISCRETE MOMENTS VERSUS $S$ DEGREE

following DT (discrete-time) structure

$$
\hat{C}(z)=\frac{r_{0}+r_{1} z^{-1}+r_{2} z^{-2}}{1+s_{1} z^{-1}+s_{2} z^{-2}}
$$

The estimated parameters are given in table III. The measured

\begin{tabular}{|c|c|c|c|c|}
\hline$r_{0}$ & $r_{1}$ & $r_{2}$ & $s_{1}$ & $s_{2}$ \\
\hline 0,02 & $-1,7 \times 10^{-3}$ & 0.011 & $-0,677$ & $-0,321$ \\
\hline
\end{tabular}

ESTIMATED PARAMETERS OF THE DT CONTROLLER

and estimated control signals are plotted in figure 10 .

\section{B. Furnace model}

The parameters are estimated using a nonlinear optimization algorithm which minimizes a quadratic criterion according to the iterative Levenberg-Marquardt algorithm [10]. The criterion is based on the output error, i.e. the error between the measured system output $y$ and the model output $\hat{y}(\underline{\hat{\theta}})$, defined by

$$
\varepsilon_{O E}=y-\hat{y}(\underline{\hat{\theta}})=y-H(s, \underline{\hat{\theta}}) u
$$

where

$$
\hat{y}(\hat{\theta})=\frac{\hat{B}(s, \hat{\theta})}{\hat{A}(s, \hat{\theta})} u(s)
$$
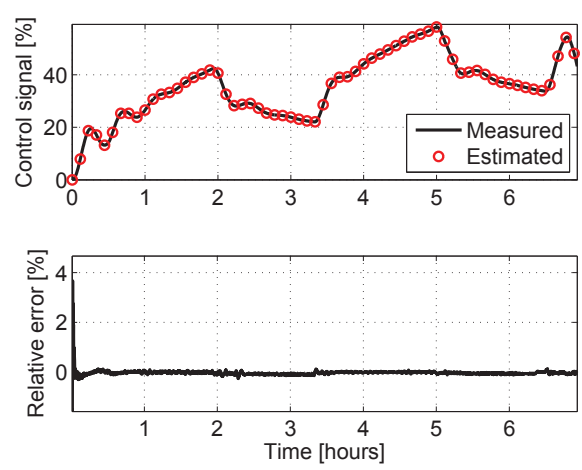

Fig. 10. Measured and estimated control signals for $S=2$

with $\hat{\theta}=\left[\hat{a}_{0}, \ldots, \hat{a}_{n_{a}-1}, \hat{b}_{0}, \ldots, \hat{b}_{n_{b}}\right]$ and $\hat{B}\left(s, \hat{\theta}_{i}\right)=\sum_{j=0}^{n_{b}} \hat{b}_{j} s^{j}, \hat{A}\left(s, \hat{\theta}_{i}\right)=\sum_{j=0}^{n_{a}-1} \hat{a}_{j} s^{j}+s^{n_{a}}$.

The Levenberg-Marquardt algorithm is defined by the following iterative equation

$$
\hat{\theta}_{i+1}=\hat{\theta}_{i}-\left\{\left[J_{\underline{\theta \theta}}^{\prime \prime}+\lambda I_{n}\right]^{-1} J_{\underline{\theta}}^{\prime}\right\}_{\theta=\hat{\theta}_{i}}
$$

where $J_{\theta}^{\prime}$ and $J_{\theta \theta}^{\prime \prime}$ are the gradient and the approximated Hessian, respectively defined by

$$
J_{\underline{\theta}}^{\prime}=-\frac{2}{K} \sum_{k=1}^{K} \varepsilon_{O E}(\underline{\hat{\theta}}) \sigma(\underline{\hat{\theta}}), \quad J_{\underline{\theta \theta}}^{\prime \prime}=\frac{2}{K} \sum_{k=1}^{K} \sigma(\underline{\hat{\theta}}) \sigma(\underline{\hat{\theta}})^{T}
$$

$\sigma(\underline{\hat{\theta}})$ is the vector of sensitivity function: $\sigma(\underline{\hat{\theta}})=\frac{\partial \hat{y}(\hat{\theta})}{\partial \underline{\hat{\theta}}}, \lambda$ is a scalar used to control the convergence, and $K$ the number of samples.

The sensitivity functions are computed as follows

$$
\sigma_{y, \hat{a}_{j}}=-\frac{s^{j} \hat{y}\left(\hat{\theta}_{i}\right)}{A\left(s, \hat{\theta}_{i}\right)}, \sigma_{y, \hat{b}_{j}}=\frac{s^{j} u\left(\hat{\theta}_{i}\right)}{A\left(s, \hat{\theta}_{i}\right)}
$$

The property of asymptotic convergence in this algorithm is achieved at the cost of the minimization of a nonlinear quadratic criteria, which can lead to a local optimum [6]. To avoid the local optimum, a solution is to use an equationerror algorithm to initialize near the global optimum. The routine IVCTRPM (Instrumental Variable Continuous-Time Reinitialized Partial Moments) can be used [11], [14]. Two different methods are compared: the OE identification method based on a closed-loop decomposition (OE-CLD) [4] and the Tailor-Made first OE method (TM) [4], [17].

1) $O E-C L D$ : We can use a DT controller with a continuoustime (CT) system as shown in Figure 11 [4]. The solution provided by the OE-CLD consists in simulating $y$ from $H(s, \underline{\hat{\theta}})$. The output sensitivity functions $\underline{\sigma}_{y, \hat{\theta}}$ should be calculated with taking account of $\underline{\sigma}_{u, \hat{\theta}}$ (the sensitivity of the predictive control signal $\hat{u}$ to $\hat{\theta}$ ).

Let us define $\underline{\sigma}_{y, \hat{\theta}_{i}}$. We know that

$$
\hat{y}(s)=H(s, \underline{\hat{\theta}}) \hat{u}(s)
$$




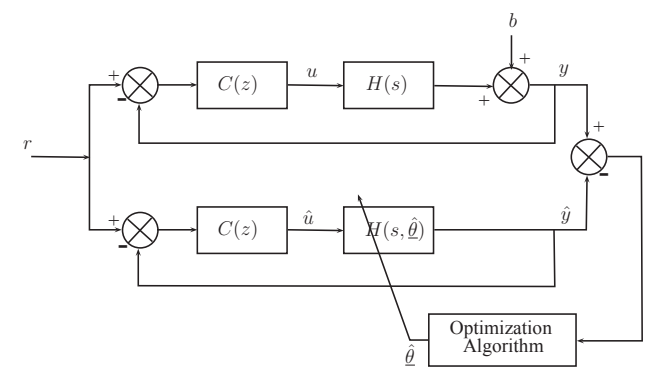

Fig. 11. OE algorithm based on a closed-loop decomposition

so

$$
\underline{\sigma}_{y, \hat{\theta}}=H(s, \underline{\hat{\theta}}) \frac{\partial \hat{u}_{k}}{\partial \hat{\theta}}+\frac{\partial H(s, \underline{\hat{\theta}})}{\partial \hat{\theta}} \hat{u}(s)
$$

with $\frac{\partial \hat{u}_{k}}{\partial \theta_{i}}$ obtained from a difference equation like (2), for example, which gives

$$
\frac{\partial \hat{u}_{k}}{\partial \hat{\theta}_{i}}+s_{1} \frac{\partial \hat{u}_{k-1}}{\partial \hat{\theta}_{i}}=-r_{0} \frac{\partial \hat{y}_{k}}{\partial \hat{\theta}_{i}}-r_{1} \frac{\partial \hat{y}_{k-1}}{\partial \hat{\theta}_{i}}
$$

in other terms

$$
\underline{\sigma}_{\hat{u}_{k}, \hat{\theta}_{i}}+s_{1} \underline{\sigma}_{\hat{u}_{k-1}, \hat{\theta}_{i}}=-r_{0} \underline{\sigma}_{y_{k}, \hat{\theta}_{i}}-r_{1} \underline{\sigma}_{y_{k-1}, \hat{\theta}_{i}}
$$

With this sensitivity function formulation, it is possible to estimate the CT system model parameters controlled by a digital controller.

2) TM: An OE method is used to globally identify the DT closed loop of the figure 12 and it was widely treated in [4], [17]. Let us consider $C(z)=\frac{R(z)}{S(z)}$ and $H(z, \underline{\hat{\theta}})=\frac{\hat{B}(z)}{\hat{A}(z)}$.

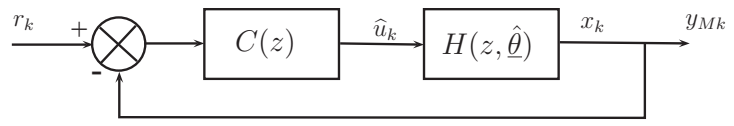

Fig. 12. DT closed-loop system

Hence

$$
y_{M}(z)=\frac{R(z) \hat{B}(z)}{S(z) \hat{A}(z)+R(z) \hat{B}(z)} r(z)
$$

or $y_{M}(z) \hat{P}(z)=R(z) \hat{B}(z) r(z)$ with

$\hat{P}(z)=[S(z) \hat{A}(z)+R(z) \hat{B}(z)]$

The calculation of the sensitivity function $\underline{\sigma}_{k}=\frac{\partial y_{M k}}{\partial \underline{\hat{\theta}}}$ gives

$$
\underline{\sigma}_{k}=\frac{S\left(q^{-1}\right)}{\hat{P}\left(q^{-1}\right)} \underline{\varphi}_{M k}
$$

with $q$ is the forward-shift time operator, and

$$
\underline{\varphi}_{M k}=\left[\begin{array}{c}
-y_{M}(k-1) \\
\vdots \\
-y_{M}\left(k-n_{a}\right) \\
\hat{u}(k-1) \\
\vdots \\
\hat{u}\left(k-n_{b}\right)
\end{array}\right]
$$

The equation (16) is used in the OE algorithm. Then, the CT transfer function $\hat{H}(s)$ is obtained from the DT one $\hat{H}(z)$.

On experimental data, we first initialize the OE algorithm with the IVCTRPM algorithm estimated in open-loop by taking as input the control signal and the measured temperature as output. The results shown in table IV are the estimated parameters of a transfer function of orders $n_{a}=2$ and $n_{b}=1$ defined by $H(s, \underline{\hat{\theta}})=\frac{G\left(1+\tau_{z} s\right)}{\left(1+\tau_{p_{1}}\right)\left(1+\tau_{p_{2}}\right)}$. FIT denotes the fitting criterion [9] which is defined by

$$
F I T=100 \times\left(1-\frac{\|y(t)-\hat{y}(t)\|}{\|y(t)-\operatorname{mean}(y(t))\|}\right)
$$

The choice of orders has been made after testing several orders. It can be seen that the IVCTRPM initialized parameters are very close to the TM and OE-CLD estimated ones. However, the difference could be more important for a more disturbed application. The fitting values show that the OE methods improve the quality of estimation. The temperature outputs are drawn in the figure 13.

\begin{tabular}{|c||c|c|c|c|c|}
\hline & $F I T[\%]$ & $G$ & $\tau_{z}$ & $\tau_{p_{1}}$ & $\tau_{p_{2}}$ \\
\hline IVCTRPM & $96 \%$ & 18,13 & 1,1329 & 0,2333 & 4,6595 \\
\hline TM & $98 \%$ & 17,56 & 0,98 & 0,23 & 4,65 \\
\hline OE-CLD & $98 \%$ & 18,37 & 1,1372 & 0,2337 & 4,9175 \\
\hline \multicolumn{7}{|c|}{ TABLE IV }
\end{tabular}

ESTIMATED PARAMETERS
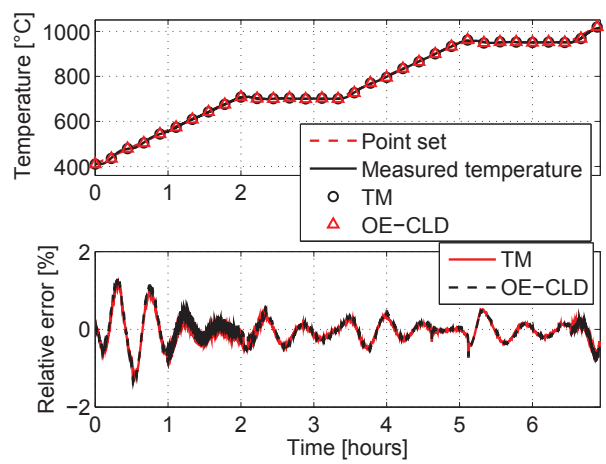

Fig. 13. Identification results

This identification is followed by a validation procedure on two other data sets by simulating the estimated closed-loop system (see Figures 14 and 15). We can easily observe that the simulated outputs are close to measurement and this result is confirmed by table $\mathrm{V}$.

\begin{tabular}{|c||c|c|}
\hline$F I T[\%]$ & TM & OE-CLD \\
\hline $2^{n d}$ test & 96.4 & 96.3 \\
\hline $3^{r d}$ test & 98.3 & 98.2 \\
\hline \multicolumn{3}{|c|}{ TABLE V } \\
VALIDATION FITTINGS
\end{tabular}

\section{Fault diagnosis results}

The furnace model is now estimated and validated. The final step consists of a diagnosis of a resistance disconnection. We have two possibilities:

- First, we can simulate the closed-loop identified below and then deduce the control signal with taking as input the setpoint temperature of the experimental data with a fault. A comparison to the measured control signal 


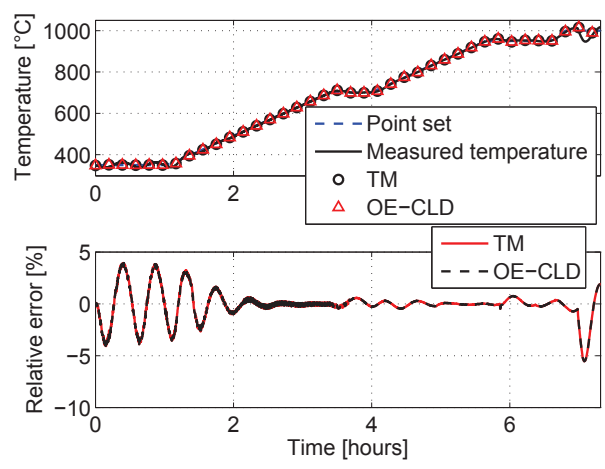

Fig. 14. Validation on a 2nd free-load test

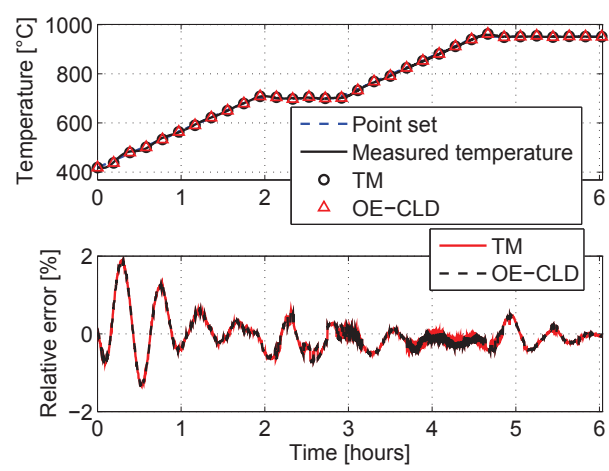

Fig. 15. Validation on a 3rd free-load test

obtained with a disconnected resistance is shown in Figure 16. It is sufficient to detect a failure and an alarm can be introduced with choosing, for example, an adaptive threshold. However, we can not distinguish what kind of failure the furnace is suffering from.

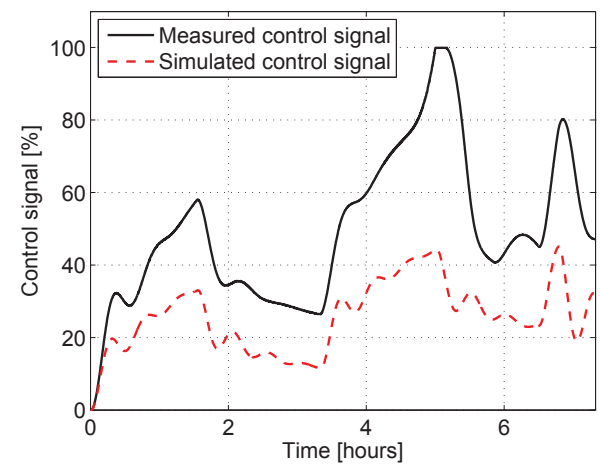

Fig. 16. Control signal divergence

- We simulate all the closed loop which includes the estimated furnace model. With this procedure, a separation can be done between a fault in the set (dimmer+resistances) (e.g. a resistance disconnection) from a fault in the furnace itself (e.g. a door opening).

\section{CONCLUSION}

In this paper, a complete study of a furnace plant was introduced with the objectives of designing a soft power sensor and of a fault diagnosis. It has also been shown that we can easily identify the model using the existing identification approaches. An indirect closed-loop estimation using two OE algorithms were investigated. For that, a previous step to estimate the controller consisting of an overparametrization technique was exploited. A successful validation is also given and the results show that the proposed methods are effective. However, only a resistance disconnection fault diagnosis was investigated but future directions as a door opening or poorly closed fault diagnosis will be explored. This diagnosis deals with building a grey box model in order to obtain a more flexible model which takes into account the mass and the load nature.

\section{ACKNOWLEDGMENT}

This work has been supported by the French Research National Agency (ANR) through Efficacité énergétique et réduction des émissions de $\mathrm{CO}_{2}$ dans les systèmes industriels program (project CHIC $\mathrm{n}^{\circ}$ ANR-10-EESI-02).

\section{REFERENCES}

[1] L. Bitschnau, M. Kozek, Modeling and control of an industrial continuous furnace, International Conference Computational Intelligence, Modelling and Simulation on Digital Object Identifier, CSSim, pp. 231 - 236, 2009.

[2] A. Chéruy, Software sensors in bioprocess engineering. Journal of Biotechnology 52, pp. 193-199, 1997.

[3] J. C. Agüro, G.C. Goodwin, P.M.J. Van den Hof, A virtual closed loop method for closed-loop identification, Automatica 47, pp. 1626-1637, 2011.

[4] O. Grospeaud, Contribution à l'identification en boucle fermée par erreur de sortie, Thesis, Université de Poitiers, Poitiers, France, 2000.

[5] O. Grospeaud and J.C. Trigeassou, Unbiased identification in closed-loop by an output error technique, European Control Conference, 1999.

[6] I.D. Landau, Unbiased recursive identification using model reference adaptive techniques, IEEE Trans. Automat. Contr., AC-21(2), pp. 194202, 1976.

[7] I.D. Landau and A. Karimi, Recursive algorithms for identification in closed-loop: A unified approach and evaluation, Automatica, Vol. 33, No 8, pp. 1499-1523, 1997.

[8] A. Le Mouel et al., Fostering Energy Efficiency in manufacturing plants through economical breakthroughs in power and flow rate measurement, ECEEE Summer Study on Industry, Arnhem, Germany, 2012.

[9] L. Ljung. System identification. Theory for the user, 2nd edition, 1999.

[10] D. Marquardt, An algorithm for least-squares estimation of nonlinear parameters. SIAM Journal on Applied Mathematics, 11, pp. 431-441.

[11] R. Ouvrard and J.C. Trigeassou, On embedded FIR filter models for identifying CT and discrete-time transfer functions: the RPM approach, Int. J. Control., 84(3), pp. 616-632, 2011.

[12] T. Poinot, Contribution à l'identification des systèmes par la méthode de surparamétrisation en traitement des eaux, Thesis, Université de Poitiers, France, 1996.

[13] M.J. Stankovski, G.M. Dimirovski, N.E. Gough, R. Hanus, Industrial furnace control: an experiment in iterative identification and design, International Conference on Control UKACC (Conf. Publ. No. 455), pp. 946-951 Vol. 2, 1998.

[14] E.Tohme, R.Ouvrard, J.C. Trigeassou, A.Abche, A pseudo-output error algorithm to improve global convergence, Conference of the IEEE Industrial Electronics Society (IECON), 2006.

[15] J.C. Trigeassou, Contribution à l'extension de la méthode des moments en automatique. Application à l'identification des systèmes linéaires, Thesis, Université de Poitiers, France, 1987.

[16] P.M.J Van den Hof, Closed-loop issues in system identification, Annual reviews in control, 22, pp. 173-186, 1998.

[17] E.T. Van Donkelaar and P.M.J. Van den Hof, Analysis of closedloop identification with a tailor-made parametrization, European Control Conference, Brussels, Belgium, Vol. 4, 1997. 